\title{
Charm rescattering contribution to charmless three-body $B$ decays
}

\section{Patricia Magalhães*t}

CBPF - Centro Brasileiro de Pesquisas Físicas

E-mail: pmagal haes@cbpf.br

\section{Bediaga}

CBPF - Centro Brasileiro de Pesquisas Físicas , Rio de Janiero - Brazil

\section{T. Frederico}

ITA - Instituto de Tecnologia e Aeronáutica, São José dos Campos - Brazil

\begin{abstract}
Charm penguin diagrams are known to be the main contribution to charmless B decay process with strangeness variation equal to minus one which is the case of $B^{ \pm} \rightarrow K^{ \pm} K^{+} K^{-}$decay. The large phase space available in this and other B three-body decays allows non trivial final state interactions with all sort of rescattering processes and also access high momentum transfers in the central region of the Dalitz plane. In this work we investigate the charm Penguin contribution to $B^{ \pm} \rightarrow K^{ \pm} K^{+} K^{-}$, described by a hadronic triangle loop in the nonperturbative regions of the phase space, and by a partonic loop at the quasi perturbative region [1]. These nonresonant amplitudes have a particular structure in the Dalitz plane and their contributions to the final decay amplitude can be confirmed by a data amplitude analysis in this channel. In particular, the hadronic amplitude has a changing sign in the phase at $D \bar{D}$ threshold which can result in a change of sign for the CP asymmetry.
\end{abstract}

XVII International Conference on Hadron Spectroscopy and Structure

25-29 September, 2017

University of Salamanca, Salamanca, Spain

\footnotetext{
${ }^{*}$ Speaker.

${ }^{\dagger}$ this work of was supported by CNPq Grant No. 157857/2015- 8.
} 


\section{Introduction}

In charmless three-body B decays the hadronic final state interactions have access to a large range of energy in the available phase space, which allow them to rescatter into other mesons. Although absent in factorization approach [2], many authors [3, 4, 5, 6, 7, 8] have shown that rescattering plays an important role in B decays. In particular, they proved the relevance for B twobody charmless decays of charm mesons rescattering into light ones, namely, in the understanding of the observed Branching fractions [5, 8] and CP violation [3, 4, 5]. It is remarkable that this rescattering contribution was never studied before within a three-body formulation.

In this work we study the contribution of a double charm intermediate interaction to the $B^{ \pm} \rightarrow K^{ \pm} K^{+} K^{-}$decay. Although this process has some suppression, the weak decay involving two charm quarks is more favourable than the one with two light quarks, which can compensate this suppression and give a significant contribution to the total decay amplitude. The $B^{ \pm} \rightarrow K^{ \pm} K^{+} K^{-}$ process is a particular interesting place to study this contribution because: (i) it has a large BR compared to other charmless three-body B decays; (ii) it is dominated by the penguin weak topology; and (iii) the experimental data from LHCb [9] showed a significant population of events spread up to high values of invariant masses.

The same LHCb paper [9] study the CP asymmetry distribution in the Dalitz plot for the four channels $B^{ \pm} \rightarrow h^{ \pm} h^{+} h^{-}(h=\pi, K)$. In particular, they showed a clear correlation between the channels $B^{ \pm} \rightarrow K^{ \pm} \pi^{+} \pi^{-}$and $B^{ \pm} \rightarrow K^{ \pm} K^{+} K^{-}$decays, observed in the region where $\pi^{+} \pi^{-} \rightarrow$ $K^{+} K^{-}$has an important contribution in the hadron-hadron scattering amplitude [10] - i.e. between 1 and $1.6 \mathrm{GeV}$. These results indicate that the rescattering process $\pi^{+} \pi^{-} \rightarrow K^{+} K^{-}$is present in these decays $[6,7]$, carrying the strong phase necessary for CP violation and conserving CPT global symmetry as discussed in Ref. [6, 7]. Analogously of what was seen for the $\pi^{+} \pi^{-} \rightarrow$ $K^{+} K^{-}$rescattering contribution to three-body charmless B decays, we investigate the hypotheses that the rescattering process $D \bar{D} \rightarrow P \bar{P}$ could provide also the strong phase needed to observe CP asymmetry in the high mass region.

\section{Charm Penguin Dynamics}

In a recent paper [11], the authors discussed the characteristics of the three-body momentum distribution along the phase space for the particular process $B^{+} \rightarrow \pi^{-} \pi^{+} \pi^{+}$. They showed that the peripheral regions of the Dalitz plot, where the light resonance are placed, are essentially nonperturbative. On the other hand, the central region of the Dalitz is dominated by large transfer momentum requiring a quasi perturbative treatment of QCD.

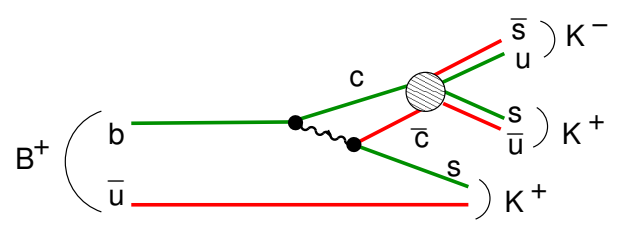

Figure 1: Penguin weak topology diagram for $B^{ \pm} \rightarrow K^{ \pm} K^{+} K^{-}$. 
Within this scenario the charm Penguin (CharmP) diagram, in Fig. 1, will contribute in distinct Dalitz regions with a different behaviour: one involving short distance physics expressed by partons loop and placed in central region; and the other one involving the long distance dynamics, which can be described by hadron loops, and are expected to be relevant in the peripheral Dalitz region. Other than give a significant contribution for the total decay rates, the CharmP can be the mechanism to explain experimental observations in charmless three-body B decays: the abundant phenomena of $\mathrm{CP}$ violation at high masses, providing the strong phase one need; and the significant population of the high mass phase space by a nonresonant amplitude.

In order to check to which extend the separation between short and long distance can be used to represent the $B^{ \pm} \rightarrow K^{ \pm} K^{+} K^{-}$decay amplitude, we investigate the two Charm Penguin contributions at the partonic and mesonic levels represented, respectively, in figures 1 and 2. Details of all the calculations can be found at [1].
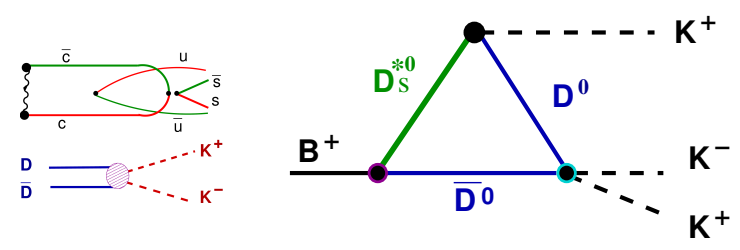

Figure 2: Left diagrams: double charm partonic loop producing $K^{+} K^{-}$(upper panel) and double charm hadronic loop producing $K^{+} K^{-}$(lower panel). Right: triangle diagram for hadronic loop for $B^{+} \rightarrow$ $K^{-} K^{+} K^{+}$with vector form factor.

\section{Partonic Charm Penguin.}

We considered the charm penguin contributions as represented by the diagram of Fig. 1 . However, is very hard to precise the effective charm mass propagating inside the loop due to the exchange of gluons and how the hadronization affects this picture. To guide our calculation one follows the structure proposed by Mannel et al. [11] to describe the center region of the Dalitz plot for $B^{+} \rightarrow \pi^{-} \pi^{+} \pi^{+}$. The authors propose a functional form of this amplitude to be $A_{p}(s)=$ $T(s)\left(M_{B}^{2}-s\right) f_{+}(s)$. Translating to $B^{+} \rightarrow K^{-} K^{+} K^{+}$process, $f_{+}\left(q^{2}\right)$ is the $B \rightarrow K$ vector form factor, which can assume the single pole parametrization: $f_{+}(s)=\frac{1}{1-s / M_{B s}^{* 2}}$, with $M_{B s}^{*}$ being the mass of a vector meson $B_{s}^{*}$. The function $T(s)$ is the kernel, which we identify as the charm parton loop. The isolate $c \bar{c}$ bubble loop contribution is very well known and we used the one calculated by Gerard and $\mathrm{Hu}(1991)[4]$.

In order to accommodate the uncertainties around charm mass, we integrate the bobble loop quark function in the charm mass convoluted with a Gaussian distribution centred in $m_{c}=1.5 \mathrm{GeV}$ and width $\Gamma=20 \mathrm{MeV}$. Those values could be taken as a free parameter when fitting real data. The final contribution to the partonic amplitude becomes [1]

$$
A_{p}^{P}=\left(M_{B}^{2}-s\right) f_{+}(s) \int_{m_{c}^{-}}^{m_{c}^{+}} d m \Pi(s) \frac{1}{2 \pi \Gamma^{2}} e^{\frac{\left(m-m_{c}\right)^{2}}{2 \Gamma^{2}}},
$$

where $m_{c}^{ \pm}=m_{c} \pm 1.0 \mathrm{GeV}$. The results for the nonresonant partonic penguin amplitude and phase are given in Fig.3. 

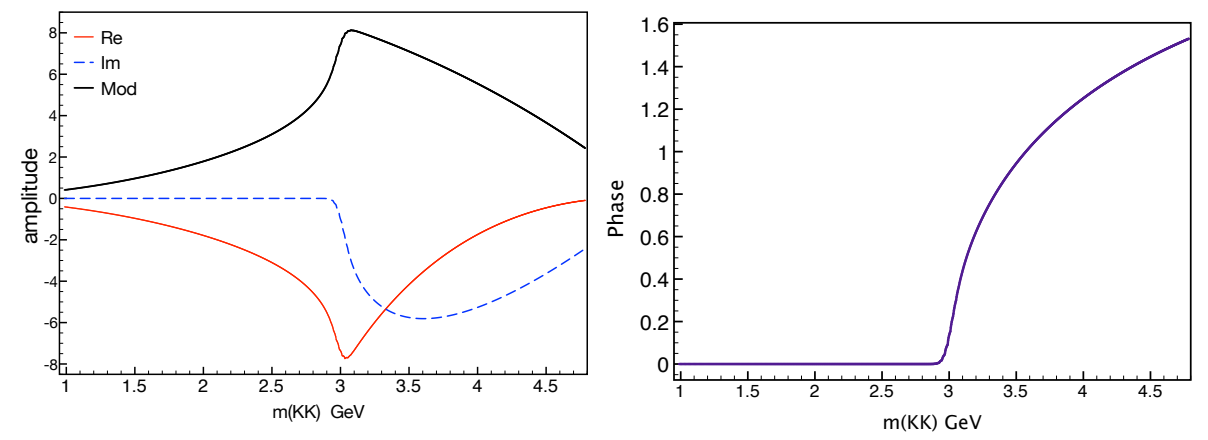

Figure 3: Modulus (left) and phase (right) of the total partonic charm Penguin amplitude, eq.(3.1).

\section{Hadronic Penguin.}

The nonresonant hadronic charm loop is expected to be important for low relative momentum between the mesons in the final state, corresponding to the boundaries of the Dalitz plot. Despite of the hadronization effect, one can expect the weak transition amplitude to be described by the diagram in the left panel of Fig.2. However, we used an effective description in terms of hadronic degrees of freedom which simplifies these interactions and are summarized by the triangle loop given in the right panel of Fig.2. It is worth to mention that there could be a superposition of similar processes with excited $D_{s}^{*}$ states, but here we are considering only the ground state $D_{s}^{+*}$ with mass $2.1 \mathrm{GeV}$.

In the triangle loop, one note that besides the weak vertex and the triangle loop itself, we need the scattering amplitude $D \bar{D} \rightarrow K \bar{K}$, which is not known in literature. Therefore, we propose a phenomenological amplitude $T_{D \bar{D} \rightarrow K \bar{K}}(s)$ based on S- matrix unitarity and inspired in Regge theory [1]. For the hadronic triangle loop we use the same technical tools find in Refs. [12, 13] developed for the three-body decays $D^{+} \rightarrow K^{-} \pi^{+} \pi^{+}$and also applied to $B^{+} \rightarrow \pi^{-} \pi^{+} \pi^{+}$[14]. The weak vertex parameters are inside the constant parameter $C_{0}$ and the transition matrix $B^{+} \rightarrow D^{0} W^{+}$is described by a form factor.

The total amplitude for the hadronic loop including the dressing of the $D \bar{D} \rightarrow K \bar{K}$ vertex by the $T_{D \bar{D} \rightarrow K \bar{K}}(s)$ scattering amplitude is given by [1]

$$
A_{P}^{h}=i C_{0} T_{D \bar{D} \rightarrow K \bar{K}}(s) \int \frac{d^{4} \ell}{(2 \pi)^{4}} \frac{\left(\Delta_{D^{0}}+2 \Delta_{\overline{D^{0}}}-2 s+3 M_{\pi}^{2}+M_{B}^{2}-l^{2}\right)}{\Delta_{D^{0}} \Delta_{\bar{D}^{0}} \Delta_{D *}\left[l^{2}-m_{B^{*}}\right]}
$$

where $\Delta_{D_{i}}=m_{D_{i}}^{2}-s+i \varepsilon$ are the meson propagators.

The final results for the magnitude and phase are show in Fig. 4. One can note that the rescattering amplitude $D \bar{D} \rightarrow K \bar{K}$ plays an important role: it imposes a zero at the $D \bar{D}$ threshold at the same place the triangle loop has a peak. Although this rescattering amplitude have parameters that needs to be fixed in a fit to data, the minimum feature is that the $D \bar{D}$ threshold is characterized by a zero between two bumps, with the higher mass one more pronounced, and is also the position where the phase changes it sign. 

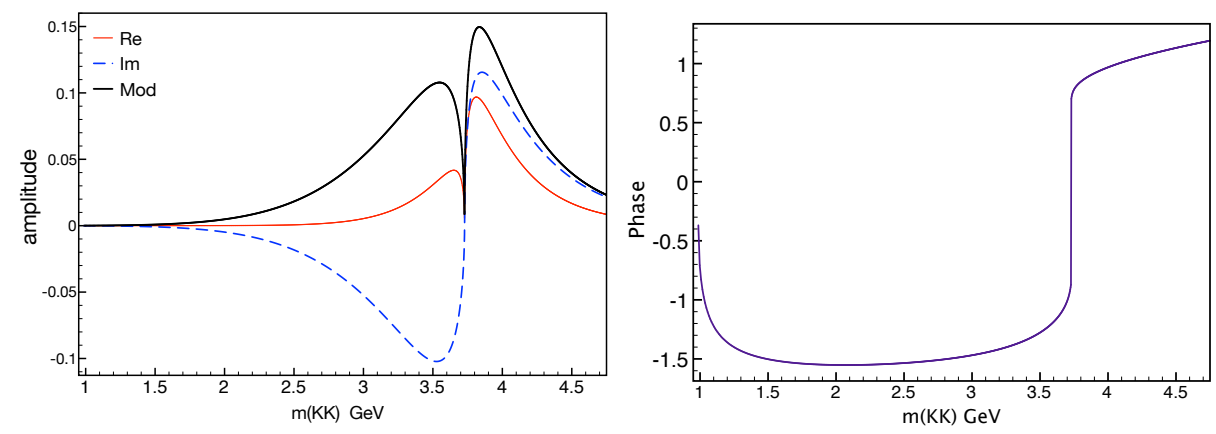

\section{Discussion.}

The structure we follow for the partonic calculation result is a wide amplitude which will be spread in the center of the Dalitz plane. This nonresonant amplitude can explain the significant number of events observed in the central region of the Dalitz plot by LHCb data[9]. The hadronic amplitude, on the other side, is characterized by two narrow peaks in between a zero at the double charm open channel.

The strong phase variation is an important signature to be observed in both charm loops. In the partonic one the phase starts at zero in the double charm threshold, around $3 \mathrm{GeV}$, and rise abruptly after that. In the hadronic one, the change of the phase sign, Fig. 4(right), is placed in a region close where data[9] shows a $\mathrm{CP}$ asymmetry change in sign $(\approx 4)$. Although we factorized the study of each charm loop, both are expected to contribute to the final amplitude. It is worth mention that we are not considering all the nonresonant nonperturbative sources. As we argue before there could be other charm hadronic triangle with heavier mesons, and also these nonresonant amplitudes are placed in a rich environment with other resonant amplitudes whose interference are not trivial. More than proving that the observed CP violation data is given by the specific hadronic loop described in Fig.4, we provide one important final state interaction (FSI) mechanism which could produce $\mathrm{CP}$ asymmetry at higher energies.

In order to evaluate our proposal, namely the relevant contribution of the hadronic loops and the partonic loop in different kinematic regions, it is important that the future amplitude analysis of the $B^{ \pm} \rightarrow K^{ \pm} K^{+} K^{-}$decay include these amplitudes in their data fits. Only then we will be able to confirm the clear separation of the relevance of partonic vs hadronic loops considering the final state interaction.

In summary, motivated by the separation of the short and long distance physics in the distribution of events in the Dalitz plane for the $B^{ \pm} \rightarrow K^{ \pm} K^{+} K^{-}$decay, we invoke a hadronic description, which we confirm that presents a very distinct pattern from the partonic one in the allowed kinematic region, driven strongly by the final state interaction amplitude, which couples the virtual intermediate double charm state to the $K^{+} K^{-}$channel, and leaving a noticeable mark in the high mass region. Such mechanism could be important to explain the CP violation observed at high mass. 


\section{References}

[1] I. Bediaga, T. Frederico, and P.C. Magalhães, ArXiV:1712.01230.

[2] M. Beneke, G. Buchalla, M. Neubert, and C. Sachrajda, Phys. Rev. Lett. 83 (1999) 1914; Nucl. Phys. B 591 (2000) 313; M. Beneke and M. Neubert, Nucl. Phys. B 675 (2003) 333.

[3] L. Wolfenstein, Phys. Rev. D 43 (1991) 151.

[4] J.-M. Gerard, W.-S. Hou, Phys. Lett. B 253 (1991) 478.

[5] H.-Y. Cheng, C.-K. Chua, A. Soni, Phys. Rev. D 71 (2005) 014030.

[6] I. Bediaga, T. Frederico, and O. Lourenço, Phys. Rev. D 89 (2014) 094013

[7] J. H. Alvarenga Nogueira, I. Bediaga, A. B. R. Cavalcante, T. Frederico and O. Lourenço, Phys. Rev. D 92 (2015) 054010.

[8] M. Gronau, D. London and J. L. Rosner, Phys. Rev. D 87 (2013) 036008.

[9] R. Aaij et al. (LHCb Collaboration), Phys. Rev. D 90 (2014) 112004.

[10] D.H. Cohen, D.S. Ayres, R. Diebold, S.L, Kramer, A.J. Pawlicki and others, Phys.Rev. D22 2595 (1980).

[11] S. Kränkl, T. Mannel and J. Virto, Nucl. Phys. B 899 (2015) 247.

[12] P. C. Magalhães, M. R. Robilotta, K. S. F. F. Guimarães, T. Frederico, W. de Paula, I. Bediaga, A. C. dos Reis, C. M. Maekawa, and G. R. S. Zarnauskas, Phys. Rev. D 84 (2011) 094001.

[13] P.C. Magalhães and M.R. Robilotta, Phys.Rev. D 92 (2015) 094005.

[14] I. Bediaga and P.C. Magalhães, arXiv:1512.09284 [hep-ph] 